\title{
Formation of Hierarchical Fuzzy Rule Systems
}

\author{
Thomas R. Gabriel and Michael R. Berthold \\ Data Analysis Research Lab \\ Tripos, Inc., 601 Gateway Blvd., Suite 720 \\ South San Francisco, CA 94080, USA. \\ Email: \{tgabriel,berthold\}@tripos.com
}

\begin{abstract}
Many fuzzy rule induction algorithms have been proposed in the past. Most of them send to generate too many rules during the learning process. This is due to data sets obtained from real world systems containing distorted elements or noisy data. Most approaches try to completely ignore outliers, which can be potentially harmful since the example may describe a rare but still extremely interesting phenomena in the data. In order to avoid this confict, we propose to build a hierarchy of fuzzy rule systems. The goal of this model-hierarchy are interpretable models with only few relevant rules on each level of the hierarchy. The resulting fuzzy model hierarchy forms a structure in which the top model covers all data explicitly and generates a significant smaller number of rules than the original fuzzy rule learner. The models on the bottom, on the other hand, consist of only a few rules in each level and explain parts with only weak relevance in the data. We demon. strate the proposed method's usefulness on several classification benchmark data sets. The results demonstrate how the rule hieranchy allows to build much smaller fuzzy rule systems and how the model-especially at higher levels of the hierarchy-remains interpretable.
\end{abstract}

\section{Introduction}

Data sets obtained from real world systems often contain missing, noisy, or simply wrong records. If one attempts to build a rule model for such data sources, the result is either an overfitted and inherently complex model, which is impossible to interpret, or the model ends up being too simplistic. ignoring most of the interesting aspects of the underlying system as well as the outliers. But even this effect of outlier ignorance is often also not desirable since the very example that was excluded from the model may be caused by a rare but still extremely interesting phenomena. The real challenge is therefore to build models that describe all inter- esting properties of the data while still being interpretable by the human expert.

First approaches to learn such hierarchical models were presented in [7]. Here an ensemble of nules sets with different granulation is built at the beginning. Starting with a coarse granulation, the remaining rule sets have increasingly finer granulation. This technique is based on a global granulation of the input space. One disadvantage is that the number of fuzzy granules increases exponentially with the level of granulation. In [4] an approach to build unsupervised hierarchical cluster models was described, however, the resulting system of clusters does not offer great interpretability.

The approach presented in this paper is based on a fuzzy rule induction algorithm presented in [3] that automatically generates fuzzy rules with only individual constraints on few attributes. The algorithm can therefore also be applied to problems of high dimensionality. The accompanying training algorithm can be used to generate a multistage fuzzy model hierarchy. The model hierarchy can be obtained through a recursive fuzzy rule induction process similar to the two-stage process introduced in [2]. At the beginning of each phase a fuzzy model is created using the remaining input pattern. A filter heuristic then extracts fuzzy rule with low relevance from the model. This filter strategy is defined based on a (subjective) measure. ment of rule importance. The extracted rules compose a so-called outlier model that describes only pattern with low relevance. The second step uses the extracted outlier rule model to filter the covered examples from the input data set. The next phase starts with the remaining examples learning a simpler fuzzy model describing only examples with higher relevance. It is interesting to see that the fuzzy models at higher hierarchical levels often show a dramatic decrease in number of rules while still achieving better generalization performance than the fuzzy rule system generated through the non-hierarchical algorithm. We evaluate the classification performance of the resulting classifierhierarchy using benchmark data sets from the European 
StatLog-Project [6].

We first briefly describe the underlying rule learning algorithm, before explaining the algorithm to construct the rule hierarchy, and conclude by discussing results on various benchmark data sets.

\section{Constructing Fuzzy Rule Models}

Extracting rule models from data is not a new area of research. In [8] and [11] algorithms were described that construct hyperrectangles in feature space. The resulting set of rules encapsulates regions in feature space that contain patterns of the same class. Other approaches, which construct fuzzy rules instead of crisp rules, were presented in $[1,5,9]$ and [10]. All of these approaches have in common that they tend to build very complex rule systems for large data sets originating from a complicated underlying system. In addition, high-dimensional feature spaces result in complex rules relying on many attributes and increase the number of required rules to cover the solution space even further. An approach that aims to reduce the number of constraints on each rule individually was recently presented in [3]. The generated fuzzy rules only constrain few of the available attributes and hence remain readable even in case of highdimensional spaces. However, this algorithm also tends to produce many rules for large, complicated data sets.

The underlying, non-hieranchical fuzzy rule learning algorithm is described in [3]. The algorithm constructs a set of fuzzy rules from given training data. The resulting set of rules can then be used to classify new data. We briefly summarize the used type of fuzzy rules before explaining the main structure of the training algorithm.

\subsection{Fuzzy Rule Systems}

The underlying fuzzy rule systems are based on a local granulation of the input space for each rule, so that rules can be independently interpreted:

$\mathcal{R}_{1}^{1}$ : IF $x_{1}$ IS $\mu_{1,1}^{1} \wedge \cdots \wedge x_{n}$ IS $\mu_{n, 1}^{1} \rightarrow$ class 1

$$
\mathcal{R}_{r_{2}}^{1}: \text { IF } x_{1} \text { IS } \mu_{1, r_{1}}^{1} \wedge \cdots \wedge x_{n} \text { IS } \mu_{n, r_{1}}^{l} \rightarrow \text { class } 1
$$

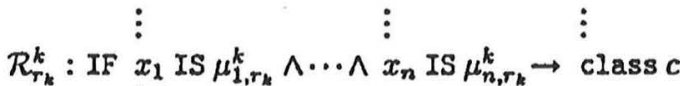

where $\mathcal{R}_{j}^{k}$ represents rule $j$ for class $k$. The rule base contains rules for $c$ classes and $r_{k}$ indicates the number of rules for class $k\left(1 \leq j \leq r_{k}\right.$ and $\left.1 \leq k \leq c\right)$.

The fuzzy sets $\mu_{i, j}^{k}: \mathbb{R} \mapsto[0,1]$ are defined for every feature $i(1 \leq i \leq n)$ and the overall degree of fulfillment of a specific rule for an input pattern $\vec{x}=\left(x_{1}, \ldots, x_{n}\right)$ can be computed using the minimum-operator as fuzzy-AND:

$$
\mu_{j}^{k}(\vec{x})=\min _{i=1, \cdots, n}\left\{\mu_{i, j}^{k}\left(x_{i}\right)\right\} .
$$

The combined degree of membership for all rules of class $k$ can be computed using the maximum-operator as fuzzy-DR:

$$
\mu^{k}(\vec{x})=\max _{j=1, \cdots, r_{k}}\left\{\mu_{j}^{k}(\vec{x})\right\} .
$$

From these membership values we then derive the predicted class $k_{\text {best }}$ for an input pattern $\vec{x}$ as:

$$
k_{\text {best }}(\vec{x})=\arg \max _{k}\left\{\mu^{k}(\vec{x}) \mid 1 \leq k \leq c\right\} .
$$

The used fuzzy membership functions are trapezoidal and can be described using four parameters $\left\langle a_{i}, b_{i}, c_{i}, d_{i}\right\rangle$, where $a_{i}$ and $d_{i}$ indicate the fuzzy rule's support- and $b_{i}$ and $c_{i}$ its core-region for each attribute $i$ of the input dimension. The training algorithm shown in the next section usually only constrains few attributes, that is, most supportregions will remain infinite leaving the rules interpretable even in case of high-dimensional input spaces.

\subsection{Construction of Fuzzy Rules}

The original algorithm [3] will be abbreviated as FRL (Fuzzy Rule Learner) in the following. During the training process all existing example patterns are subsequentially presented to the growing model. The algorithm then introduces new fuzzy rules when necessary and adjusts the coverage (support- and core-regions) of already existing rules in case of conflicts. The complete training data is presented once during each epoch. For each training pattern three different steps are executed:

- Cover: If a new training pattern lies inside the supportregion of an already existing fuzzy rule of the correct class, its core-region is extended to cover the new pattern. In addition, the weight of this rule is incremented.

- Commit: If the new pattern is not yet covered, a new fuzzy rule belonging to the corresponding class will be created. The new example is assigned to its coreregion, whereas the overall rule's support-region is initialized "infinite", that is, the new fuzzy rule is unconstrained and covers the entire domain.

- Shrink: If a new pattern is incorrectly covered by an existing fuzzy rule of conflicting class, this fuzzy rule's support-region will be reduced, so that the conflict with the new pattern is avoided. The underlying heuristic of this step aims to minimize the loss in volume (see [3] for details).

The algorithm usually terminates after only few iterations over the training data. The final set of fuzzy rules can be used to compute a degree of class membership for new input patterns as described in Section 2.1. 


\section{Learning Hierarchies of Rule Systems}

The fuzzy rule learner described in the previous section has the usual problems when encountering noisy data or outliers. In such cases an excessive number of rules is being introduced simply to model noise and outliers. This is due to the fact that the algorithm aims to generate conflict free rules, that is, examples encountered during training will result in a non-zero degree of membership only for rules of the correct class. Unfortunately, in case of noisy data, which distorts class boundaries, such an approach will result in many superfluous mules introduced simply to model these artifacts.

Using an already existing model we can, however, easily determine these parts of a rule model since they tend to have very low relevance. The main idea behind the hierarchical training algorithm is to use those parts of the rule model with low relevance as a filter for the input data, which in turn is used to create the next layer. This recursive process of data cleansing results in higher levels of the hierarchy, which are built only for examples with strong support in the original data set. For this to work, we obviously need to define the notion of rule relevance or importance more precisely.

\subsection{Filtering Outlier Rules}

To measure a rule's relevance often the weight parameter $\omega^{(\mathcal{R})}$ is used, which represents the number of training patterns covered by rule $\mathcal{R}$. A measure for the importance or relevance of each rule can be derived by simply using the percentage of patterns covered by this rule:

$$
\Phi(\mathcal{R})=\frac{\omega^{(\mathcal{R})}}{|\mathbf{T}|} .
$$

where $\mathrm{T}$ indicates the set of all training examples.

The choice of relevance metric is made depending on the nature of the underlying rule generation algorithm, as well as the focus of analysis, i. e. the interpretation of important vs. unimportant or irrelevant data points.

Using any of the above-mentioned measures of (notably subjective) relevance, together with a relevance threshold $\theta_{\text {outlier, }}$ we can now extract rules with low relevance from the model:

$$
\mathbf{R}^{\text {outlier }}=\left\{\mathcal{R} \in \mathbf{R} \mid \Phi(\mathcal{R}) \leq \theta_{\text {outlier }}\right\}
$$

with $\mathbf{R}=\bigcup_{k=1}^{c} \mathbf{R}^{k}\left(\mathbf{R}^{k}=\left\{\mathcal{R}_{1}^{k}, \ldots, \mathcal{R}_{r_{k}}^{k}\right\}\right)$ for all rules of class $k$.

Using this outlier model as a filter for a second training phase will then generate a new fuzzy model, which has fewer rules with higher significance. In effect, the orig. inal training data is filtered and only data points which are not covered by the outlier model are passed onto the next level for training ${ }^{l}$. For thiṣ a filter-threshold $\theta_{\text {filter }}$ $\left(0 \leq \theta_{\text {filter }} \leq 1\right)$ is used:

$$
\begin{aligned}
\mathbf{T}^{\text {relevant }}= & \mathbf{T} \backslash\{(\vec{x}, k) \in \mathbf{T} \mid \\
& \left.\exists \mathcal{R} \in \mathbf{R}^{\text {outlier }}: \mu_{\mathcal{R}}(\vec{x}) \geq \theta_{\text {filter }}\right\} .
\end{aligned}
$$

This procedure was used in [2] to remove potential outliers from the training data. There, the initial model is used to extract an outlier model, which is subsequentially used to filter the data and generate the final rule model.

\subsection{Constructing Rule Hierarchies}

An entire model hierarchy can be obtained by a multistage fuzzy rule induction process based on the two-stage procedure described above. This hierarchical training process recursively continues to apply the filtering procedure until a final rule model is generated, where all rules lie above the relevance threshold. The resulting hierarchical Fuzzy Rule Learner (or hFRL) can then formally be described as shown in Table 1.

After some initializations, line (4) shows the generation of the fuzzy rule model using the original FRL for the input data separately for each level of the hierarchy. From the resulting rule set, we extract rules with low relevance using the outlier-threshold $\theta_{\text {outlier }}$ at line (5). The next step extracts all examples from the rule set using the filter parameter $\theta_{\text {filter. }}$. This procedure is repeated until all rules are above the outlier-threshold and the outlier model remains empty. The model hierarchy consists of all outlier models $\mathbf{R}_{i}$ and the rule model of the last iteration, which we denote by $\boldsymbol{R}_{x}$.

Figure 1 shows a two-dimensional example with patterns of two classes (cross and circle). The rectangles depict the rule's core-regions only. In this example the three-level hierarchy is built using an outlier-threshold $\theta_{\text {outlier }}=2$ and a filter-threshold $\theta_{\text {filter }}=1.0$. That is, rules covering two or

\begin{tabular}{|c|c|}
\hline (1) & $T_{0}=T$ \\
\hline (2) & $i \leftarrow 0$ \\
\hline (3) & REPEAT \\
\hline (4) & $\mathbf{R}_{i}=\operatorname{FRL}\left(\mathbf{T}_{\mathbf{i}}\right)$ \\
\hline (5) & $\mathbf{R}_{i}^{\text {outlier }}=\left\{\mathcal{R} \in \mathbf{R}_{i} \mid \Phi(\mathcal{R}) \leq \theta_{\text {outlier }}\right\}$ \\
\hline (6) & $\begin{array}{l}\mathbf{T}_{i+1}=\mathbf{T}_{\boldsymbol{i}} \backslash\left\{(\vec{x}, k) \in \mathbf{T}_{\mathbf{i}} \mid\right. \\
\left.\quad \exists \mathcal{R} \in \mathbf{R}_{i}^{\text {outlier }}: \mu_{\mathcal{R}}(\vec{x}) \geq \theta_{\text {filter }}\right\}\end{array}$ \\
\hline (7) & $i \leftarrow i+1$ \\
\hline (8) & WHILE $\left|R_{i-1}^{\text {outlier }}\right|>0$ \\
\hline
\end{tabular}

'Note that this procecture is similar to well-known pruning strategies. In this case, however, the pruned model parts are not discarded but instead used as explicit outlier model. 


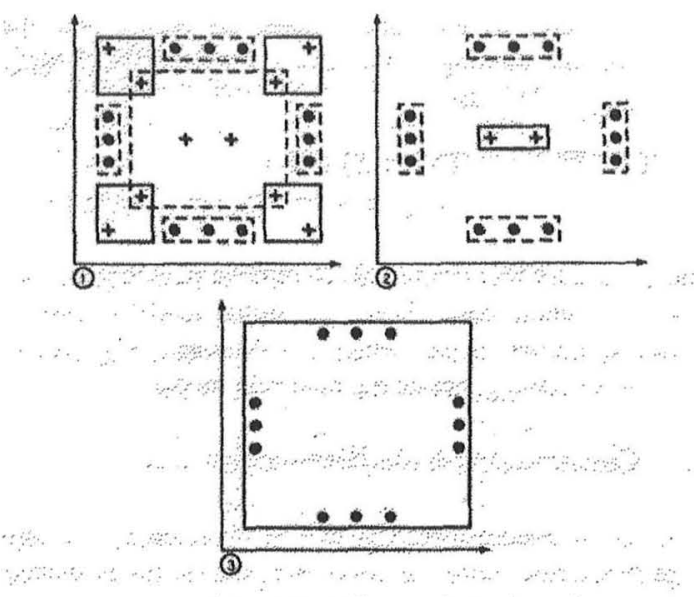

Figure 1. An example rule hierarchy, using the original rule-based filtering strategy.

less patterns are moved to the outfier model and only patterns falling inside the core-region of an outlier rule (hence resulting in a degree of membership of at least one) are removed.

Figure 1(1) illustrates the original rule model consisting of nine rules generated at the beginning of the process. The four rules in the cormers cover only two patterns each and are therefore added to the first outlier model. The remaining five rules (dashed lines) are not used at this level. The algorithm then removes the corresponding patterns from the training data to be used at the next level. Figure I(2) displays the next model, containing five rules. Here, only the rule in the center does not cover more than two example and composes the second outlier model. The last step generates the final model with one rule covering all remaining patterns of class circle (Figure 1(3)).

The example in Figure $I$ shows how this rule-based filtering mechanism can potentially remove large portions of the training data during subsequent stages. This is due to
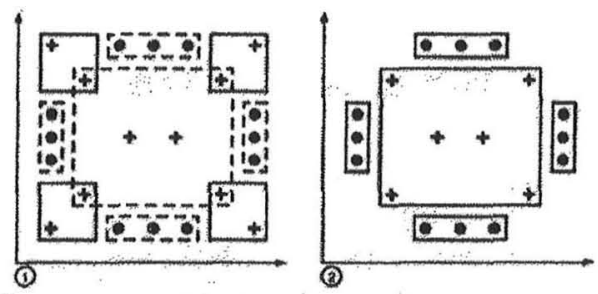

Figure 2. An example of a model-based rule hierarchy, using the same data as in Figure 1. the fact that only the individual rule's coverage of patterns is considered. More reasonable results can be achieved using a global, model-based approach.

Our filter criterion can easily be extended accordingly. In addition to the test in line (6) if an outlier nule exists that covers a certain pattern, we now also ensure that no other non-outlier rule covers this pattern:

$$
\begin{aligned}
\mathbf{T}_{i+1}= & \mathbf{T}_{\boldsymbol{i}} \backslash\left\{(\vec{x}, k) \in \mathbf{T}_{i} \mid\right. \\
& \exists \mathcal{R} \in \mathbf{R}^{\text {outlier }}: \mu_{\mathcal{R}}(\vec{x}) \geq \theta_{\text {filter }} \wedge \\
& \left.\nexists \mathcal{R} \in \mathbf{R}_{i} \backslash \mathbf{R}_{i}^{\text {outlier }}: \mu_{\mathcal{R}}(\vec{x}) \geq \theta_{\text {filter }}\right\} .
\end{aligned}
$$

Figure 2 demonstrates the impact of this change using the same data as before. Figure 2(1) again shows the first model in the hierarchy. The rules in the corners are again added to the first outlier model. But this time, the inner data points of these rules are also covered by the rule in the center, which is not part of the outlier model. Therefore these points remain in the training data for the next iteration. The final model in Figure 2(2) contains five rules, all of which are above the outlier-threshold.

This model-based approach enables us to only remove patterns from the next stage training data that are solely covered by rules of the outlier model and avoids the overly pessimistic problem demonstrated above.

\subsection{Classification using Hierarchies}

In Section 21 we showed how a non-hierarchical rule system can be used for classification of new input patterns, resulting in a set of fuzzy membership values for each class. In case of a hierarchical rule system, the outputs of the rule systems at different levels need to be combined.

Figure 3 shows an example using the hierarchical fuzzy rule model of Figure 1. Each rule model, $\mathbf{R}_{0}, \mathbf{R}_{1}$, and $\mathbf{R}_{x}$, provides its own output fuzzy membership degrees for a given input pattem. For the displayed rule system, $R_{0}$ does not make a prediction for the given example, $R_{1}$ outputs class cross and $\mathbf{R}_{x}$ predicts class circle. For our tests we use two classification strategies. One accumulates the fuzzy membership degrees of each level by summing them (FuzzySum $\mathbf{R}_{r} \rightarrow \mathbf{R}_{i}$ ), the other one (FirstHit $\mathbf{R}_{i}\left[\rightarrow \mathbf{R}_{x}\right.$ ) determines the output based on the model-level that covers the pattern first, using a bottom-up strategy.

In the following section we report experimental results using varying depths of the hierarchy. That is, we use the top level model along with an increasing number of outlier models for classification.

\section{Experimental Results}

Eight benchmark data sets from the StatLog-Project [6] are used to demonstrate the performance of the proposed 


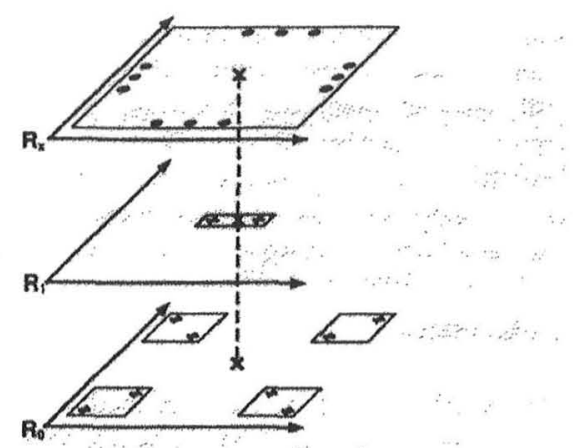

Figure 3. Classification of a new pattern using a three-level rule hierarchy.

methodology. We follow the division in training/test set resp. number of cross validation folds as specified in [6] For the tests we choose an outlier-threshold $\theta_{\text {outlier }}=5$, that is, rules which cover less than five pattern are moved to the outlier model. We ran preliminary experiments with various choices for this threshold, and this choice seems to be an appropriate value to generate an acceptable depth of the hierarchy for all data sets.

The following paragraphs discuss the results of two of the eight data sets in more detail. In each of the corresponding diagrams, the graph on top shows the error of the nonhierarchical fuzzy rule learner (dashed line) and the performance curves of both classification strategies introduced above. For those two cases, the number of outlier models used for classification was varied (left: using all models down to $\mathbf{R}_{0}$; right: using only the top level model $\mathbf{R}_{x}$ ). In addition, the graph on the bottom shows the number of rules generated by the original fuzzy rule learner ( $\mathrm{R}$ ), as well as the number of rules in each level $\mathbf{R}_{i}$ to $\mathbf{R}_{x}$ of the hierarchy. At the end of this section we summarize the results on these and the remaining five data sets.

\subsection{Vehicle Silhouette Data Set}

Figure 4 illustrates the results on the Vehicle Silhouette data set. The proposed method generates 8 hierarchy levels using the training data. The classical fuzzy model consists of 165 rules producing an error of $33.0 \%$ on the test data. Using only the nule base at the top level $\left(\mathbf{R}_{x}\right)$ a similar performance $(33.5 \%)$ can be achieved with only 49 rules: Slightly better performance $(32.7 \%)$ results from using FuzzySum and models $\mathbf{R}_{x}$ to $\mathbf{R}_{4}$ with 57 rules in total. Subsequentially adding models decreases this error further to $31.6 \%$ with altogether 186 rules. On the other hand, using the FirstHit-strategy and all levels of the generated hierarchy produces substantially worse classification results.

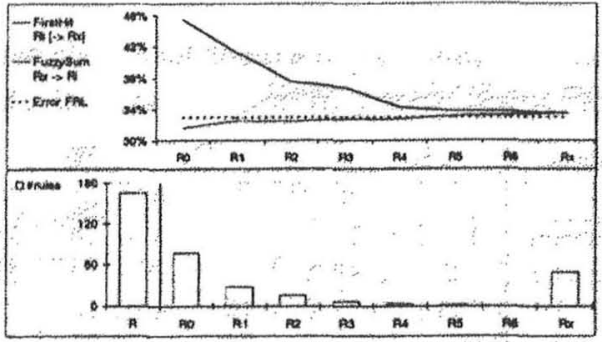

Figure 4. Vehicle Silhouette data $(752$ patterns, 8-level hierarchy).

However, this error declines quickly, when the lower few levels of hierarchy are ignored. This indicates that these parts of the model hierarchy represent artifacts in the training data that are not representative for the underlying concept.

\subsection{Australian Credit Approval}

Experiments on the the Australian Credit Approval with 690 patterns are shown in Figure 5. The non-hierarchical fuzzy rule learning algorithm generates 125 rules with an error of $18.8 \%$ on the test data. The hierarchical rule learner with 5 levels achieves a noticeably better performance of $15.4 \%$ using only 31 rules at the top level. The entire rule hierarchy consists of 91 niles with a minimum error rate of $15.2 \%$. Again, note how when using the FirstHitstrategy the addition of the first outlier model $\boldsymbol{R}_{0}$ signifcantly increases the generalization error, indicating a substantial amount of artifacts in the original training data, which were filtered out during the first phase of training.

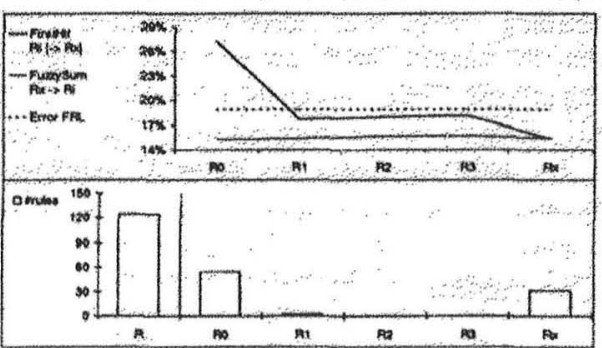

Figure 5. Australian Credit Approval (690 patterns, 5 -level hierarchy). 
Table 2. Summary of the result for all data sets from the StatLog-Project.

\begin{tabular}{|l||l|l||l|l|l|l|l|}
\hline \multicolumn{1}{|c||}{ Data set } & \multicolumn{2}{c|}{$\mathbf{R}$} & \multicolumn{2}{c|}{$\mathbf{R}_{\boldsymbol{x}}$} & \multicolumn{2}{c|}{$\mathbf{R}_{x} \rightarrow \mathbf{R}_{0}$} \\
& $|\mathcal{R}|$ & error & $|\mathbf{R}|$ & $|\mathcal{R}|$ & error & $|\mathcal{R}|$ & error \\
\hline \hline Diabetes & 151 & 32.3 & 11 & $\mathbf{5 6}$ & $\mathbf{2 6 . 0}$ & 162 & $\mathbf{2 8 . 4}$ \\
Aust. Cr. & 125 & 18.8 & 5 & $\mathbf{3 1}$ & $\mathbf{1 5 . 4}$ & $\mathbf{9 1}$ & $\mathbf{1 5 . 2}$ \\
Vehicle & 165 & 33.0 & 8 & $\mathbf{4 9}$ & 33.5 & 186 & $\mathbf{3 1 . 6}$ \\
Segment & 96 & 3.9 & 6 & $\mathbf{7 0}$ & 4.9 & 96 & 4.5 \\
Shuttle & 60 & 0.06 & 3 & $\mathbf{3 0}$ & 0.15 & $\mathbf{5 7}$ & 0.09 \\
Satlmage & 416 & 13.8 & 14 & $\mathbf{1 3 2}$ & $\mathbf{1 4 . 0}$ & 490 & $\mathbf{1 3 . 2}$ \\
DNA & $\mathbf{7 4 2}$ & 32.7 & $\mathbf{9}$ & $\mathbf{1 5 8}$ & $\mathbf{4 4 . 9}$ & 850 & 39.6 \\
Letter & $\mathbf{2 3 6 9}$ & $\mathbf{1 4 . 4}$ & $\mathbf{1 3}$ & $\mathbf{7 1 4}$ & $\mathbf{2 3 . 0}$ & $\mathbf{3 2 5 2}$ & $\mathbf{1 4 . 8}$ \\
\hline
\end{tabular}

\subsection{Summary Results}

Table 2 summarizes the results of all eight benchmark data sets from the StatLog-Project [6] used for our experiments. The table shows the results in comparison to the non-hierarchical fuzzy rule learner.

The table is separated into three parts. The first column shows the name of the data set, followed by the results of the FRL algorithm (R). Column (2) and (3) list the corresponding number of rules and error rate in percent. The next column shows the number of generated rule levels, followed by the number of rules and the performance of the top model in the hierarchy $\left(\mathbf{R}_{x}\right)$. Finally, the last two columns show the number of rules and the classification error $\left(R_{x} \rightarrow R_{0}\right)$ of the entire hierarchy using the FuzzySum-strategy.

Note how for all eight data sets the number of rules at the top level of the hierarchy is substantially smaller than in the original model. For the Pima Indians Diabetes data base and Australia Credit Approval an even better performance can be achieved using just the top level model. Often even the entire hierarchy is smaller than the classical nonhierarchical rule system (e.g. Australia Credit Approval and Shuttle data set). This cleariy indicates that the hierarchical rule learning strategy does indeed extract rules-and subsequentially patterns-with low relevance throughout the training process. The results on the DNA data are not representative since the feature space consists of 240 binary variables. The underlying fuzzy rule learner is highly unstable in this case.

\section{Conclusion}

We have described a method that allows to build a hierarchy of rules based on an underlying, non-hierarchical rule learning algorithm. Using several benchmark data sets we showed how the classification accuracy was comparable and sometimes even better than the underlying, non-hierarchical algorithm and how the resulting rule systems are of substantially reduced complexity. Due to the general nature of the method proposed here, it is possible to apply it to other local learning methods. As long as it is possible to extract parts of the model with low relevance, those pieces can then be used as a filter for subsequent levels in the hierarchy.

\section{References}

[1] S. Abe and M.-S. Lan. A method for fuzzy rules extraction directly from numerical data and its application to pattern classifiction. IEEE Transactions on Fuzzy Systems, 3(1):18-28, 1995.

[2] M. R. Berthold. Leaming fuzzy models and potential outliers. In Computational Intelligence in Data Mining, pages 111-126. Springer-Verlag, 2000.

[3] M. R. Berthold. Mixed fuzzy rule formation. International Journal of Approximate Reasoning (IJAR), 32:67-84, 2003.

[4] A. B. Geva. Hierarchical unsupervised fuzzy clustering. IEEE Transactions on Fuzzy Systems, 7(6):723733, Dec. 1999.

[5] C. M. Higgins and R. M. Goodman. Learning fuzzy rule-based neural networks for control. In Advances in Neural Information Processing Systems, 5, pages 350-357, California, 1993. Morgan Kaufmann.

[6] D. Michie, D. J. Spiegelhalter, and C. C. Taylor, editors. Machine Learning, Neural and Statistical Classification. Ellis Horwood Limited, 1994.

[7] K. Nozaki, H. Ishibuchi, and H. Tanaka. Adaptive fuzzy rule-based classification systems. IEEE Transactions on Fuzzy Systems, 4(3):238-250, 1996.

[8] S. Salzberg. A nearest hyperrectangle learning method. In Machine Learning, 6, pages 251-276, 1991.

[9] P. K. Simpson. Fuzzy min-max neural networks - part 1: Classification. IEEE Transactions on Neural Networks, 3(5):776-786, Sept. 1992.

[10] L.-X. Wang and J. M. Mendel. Generating fuzzy rules by learning from examples. IEEE Transactions on Systems, Man, and Cybernetics, 22(6):1313-1427. 1992.

[11] D. Wettschereck. A hybrid nearest-neighbour and nearest-hyperrectangle learning algorithm. In Proceedings of the European Conference on Machine Learning, pages 323-335, 1994. 